\title{
Sequential Therapy versus Triple Therapy for the First Line Treatment of Helicobacter pylori in Korea: A Nationwide Randomized Trial
}

\author{
Joon Sung Kim ${ }^{1}$, Byung-Wook Kim ${ }^{1}$, Su Jin Hong ${ }^{2}$, Jin II Kim ${ }^{3}$, Ki-Nam Shim ${ }^{4}$, Jie-Hyun Kim ${ }^{5}$, Gwang Ho Baik ${ }^{6}$, Sang Wook \\ $\mathrm{Kim}^{7}$, Hyun Joo Song ${ }^{8}$, and Ji Hyun Kim ${ }^{9}$ \\ ${ }^{\prime}$ Department of Internal Medicine, Incheon St. Mary's Hospital, The Catholic University of Korea College of Medicine, Incheon, ${ }^{2}$ Department of \\ Internal Medicine, Soonchunhyang University College of Medicine, Bucheon, ${ }^{3}$ Department of Internal Medicine, Yeouido St. Mary's Hospital, \\ The Catholic University of Korea College of Medicine, Seoul, ${ }^{4}$ Department of Internal Medicine, Ewha Medical Research Institute, Ewha \\ Womans University School of Medicine, Seoul, ${ }^{5}$ Department of Internal Medicine, Yonsei University College of Medicine, Seoul, ${ }^{6}$ Department of \\ Internal Medicine, Hallym University College of Medicine, Chuncheon, ${ }^{7}$ Department of Internal Medicine, Chonbuk National University Medical \\ School, Jeonju, ${ }^{8}$ Department of Internal Medicine, Jeju National University School of Medicine, Jeju, and ${ }^{9}$ Department of Internal Medicine, \\ Inje University Busan Paik Hospital, Inje Univeristy College of Medicine, Busan, Korea
}

Background/Aims: Eradication of Helicobacter pylori infection with standard triple therapy (TT) has declined primarily because of increased antibiotic resistance. Sequential therapy (ST) has been suggested as an alternative to $\pi$ for the first-line treatment of $\mathrm{H}$. pylori. The purpose of this study was to compare the efficacy of ST with TT. Methods: This was a multicenter, randomized open-label trial performed at nine centers in Korea. Patients with $H$. pylori infection were randomly assigned to receive either 7 day TT or 10 day ST. Eradication rates, drug compliance, and adverse events were compared among the two regimens. Results: A total of 601 patients were enrolled between March 2011 and September 2014. The intention-to-treat eradication rates were $70.8 \%$ for TT and $82.4 \%$ for ST ( $p=0.001$ ). The corresponding per protocol eradication rates were $76.9 \%$ and $88.8 \%$ for $\Pi$ T and ST, respectively ( $p=0.000)$. There were no statistically significant differences between the two regimens with respect to drug compliance and adverse events. Conclusions: ST achieved better eradication rates than $\Pi$ as a first-line therapy for $H$. pylori eradication in Korea. (Gut Liver 2016;10:556-561)

Key Words: Helicobacter pylori; Drug resistance; Disease eradication

\section{INTRODUCTION}

Helicobacter pylori is a known cause of gastritis, peptic ulcer, mucosa-associated lymphoid tissue lymphoma (MALToma), and gastric cancer. ${ }^{1,2}$ Eradication of $H$. pylori is essential for the treatment of these diseases and is currently recommended in numerous guidelines. ${ }^{3-6}$ Most international guidelines recommended triple therapy (TT) regimens consisting of a proton pump inhibitor (PPI), clarithromycin, and amoxicillin/metronidazole for at least 7 days for eradication of H. pylori. ${ }^{7-10}$ However the eradication rates of TT has decreased to unacceptable levels of less than $80 \%$ in the past decade due to increased $H$. pylori resistance to antibiotics. ${ }^{11,12}$ Although the seroprevalence rate of $H$. pylori has been reported to be decreasing in Korea, it is still very common infecting more than half of the adult population. $^{13}$

The TT regimen is recommend as first line therapy in the recent Korean guidelines and is the only regimen reimbursed by the Korean National Health Insurance Service (NHIS). ${ }^{14}$ However, the eradication rates of TT has been reported to be declining in Korea similar to the trend shown in other countries. ${ }^{15,16}$

The sequential therapy (ST) consists of a PPI and amoxicillin for the first 5 days followed by a PPI plus clarithromycin and metronidazole (or tinidazole) for the following 5 days. ${ }^{17}$ The ST regimen has been suggested as an alternative first line regimen and several systematic reviews have found the ST to be superior to TT for eradication of $H$. pylori. ${ }^{18-21}$ However, clarithromycin resistance is reported to be as high as $37.3 \%$ in Korea. ${ }^{22}$ A metaanalysis found that although ST achieved higher eradication rates than the TT regimens, its eradication rate was lower in Korea compared with previous reports. ${ }^{23}$ However, most of the studies included in this meta-analysis were either single center

Correspondence to: Byung-Wook Kim

Division of Gastroenterology, Department of Internal Medicine, Incheon St. Mary's Hospital, The Catholic University of Korea College of Medicine, 56 Dongsu-ro, Bupyeong-gu, Incheon 21431, Korea

Tel: +82-32-280-5051, Fax: +82-32-280-5987, E-mail: gastro@catholic.ac.kr

Received on September 17, 2015. Revised on October 28, 2015. Accepted on October 28, 2015. Published online April 28, 2016 pISSN 1976-2283 eISSN 2005-1212 http://dx.doi.org/10.5009/gnl15470

@ This is an Open Access article distributed under the terms of the Creative Commons Attribution Non-Commercial License (http://creativecommons.org/licenses/by-nc/4.0) which permits unrestricted non-commercial use, distribution, and reproduction in any medium, provided the original work is properly cited. 
studies or locoregional studies. Thus, we decided to perform a nationwide study comparing the eradication rates of ST with TT to find out if ST can replace TT as first line therapy in Korea

\section{MATERIALS AND METHODS}

\section{Study design and population}

This was a multicenter, randomized, open-label, and doublearm study. Patients with confirmed $H$. pylori infection were enrolled from nine tertiary hospitals from March 2011 to September 2014 (Fig. 1). Currently, the NHIS reimburses eradication therapy for patients diagnosed with peptic ulcer disease or gastric MALToma. Consecutive patients (19 to 75 years of age) who had received endoscopy at each hospital and who met the criteria for eradication by NHIS were candidates for inclusion in the study. Patients who had received endoscopic treatment for early gastric cancer were also included in our study. Exclusion criteria included use of PPI, $\mathrm{H}_{2}$-receptor antagonist, bismuth preparation and antibiotics 2 weeks before enrollment, prior eradication treatment, history of gastrectomy, and presence of severe comorbidities or malignancy. Patients deemed inappropriate for inclusion by the investigator were excluded, as were patients who refused to participate in the study. All included patients provided written informed consent prior to their participation. The study was approved by Korean Ministry of Drug and Food Safety (11930) along with the ethics committees at each participating hospital. This study was registered at Clinical Research Information Service (КСТ0000378).

\section{Detection of $\boldsymbol{H}$. pylori infection and confirmation of erad- ication}

All patients underwent upper gastrointestinal endoscopy before enrollment in this study. $H$. pylori infection was defined as positive if the results of one of the following tests were positive: histological assessment of $H$. pylori by modified Giemsa stain according to the Sydney system, and rapid urease test using samples from the antrum and distal corpus. H. pylori eradication was evaluated by the two tests used for diagnosis or by ${ }^{13} \mathrm{C}$-urea breath test 4 weeks after completion of treatment.

\section{Randomization and intervention}

Patients were randomly assigned to two treatment groups in a 1:1 ratio using a computerized random sequence when the patient agreed to participate in this study. The TT group received a full dose PPI such as lansoprazole $30 \mathrm{mg}$ or pantoprazole 40 $\mathrm{mg}$, amoxicillin $1 \mathrm{~g}$, and clarithromycin $500 \mathrm{mg}$ twice daily for 7 days. The ST group received a full dose PPI and amoxicillin $1 \mathrm{~g}$ twice daily for 5 days, followed by a full dose PPI, clarithromycin $500 \mathrm{mg}$, and metronidazole $500 \mathrm{mg}$ twice daily for 5 days. Compliance and adverse events were assessed by interview after the end of the treatment.

\section{Measurements and outcomes}

The primary endpoint of the study was $H$. pylori eradication rate in the ST group compared to the TT groups. Secondary endpoints were adverse effects and compliance to treatment in the TT and ST groups.

\section{Statistical analysis}

The eradication rate of ST and conventional TT was assumed to be $80 \%$ and $70 \%$, respectively. ${ }^{23}$ The sample size needed to detect a difference of $10 \%$ in the eradication between the two regimens with a power of $80 \%$ and a significance level of 0.05 was calculated. A drop-out rate of $20 \%$ was anticipated. The final calculated sample size was 290 patients for each group. For intention-to-treat (ITT) analysis, all patients who took the

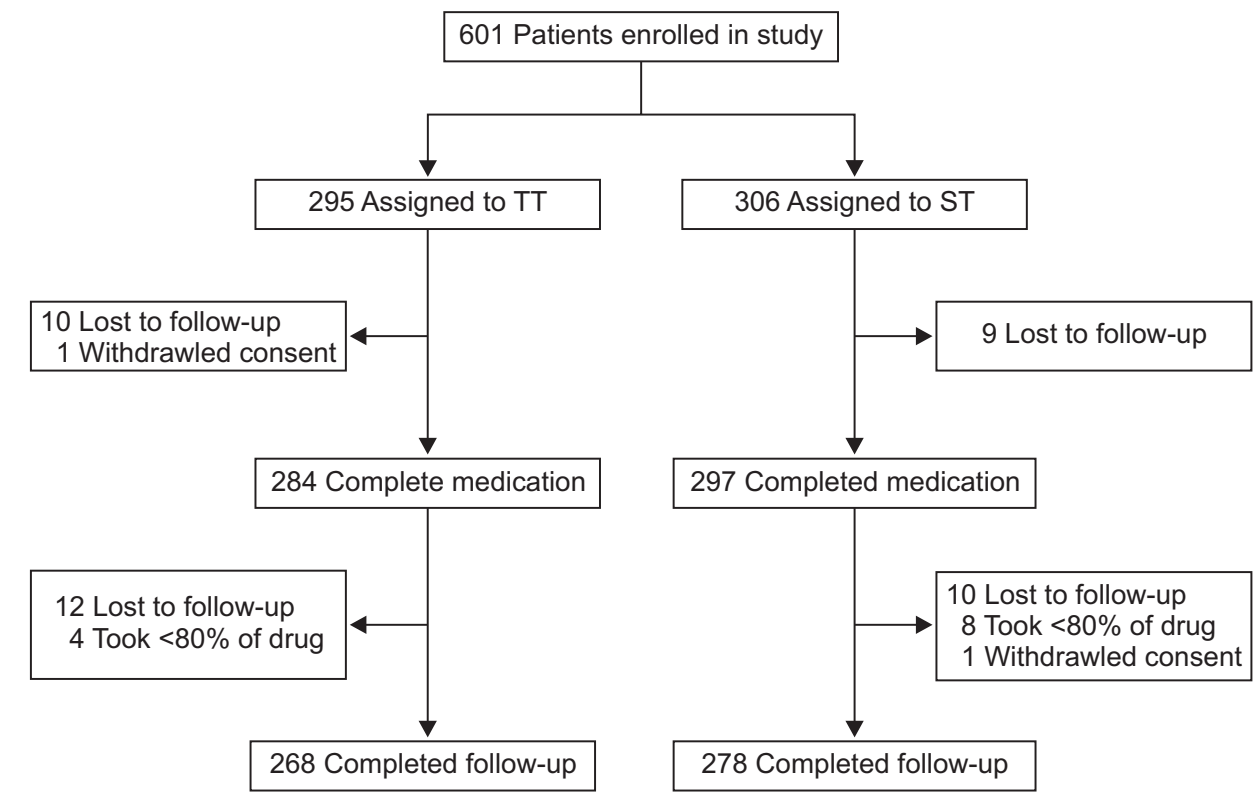

Fig. 1. Flow diagram of the participants included in the study. TT, triple therapy; ST, sequential therapy. 
prescription for eradication therapy were included. For the per protocol (PP) analysis, patients who had taken $<80 \%$ of their medication were excluded as well as patients who did not return for follow-up. Chi-square and Student t-tests were used to compare proportions and means for normally distributed data. For all tests, a p-value $\leq 0.05$ was considered as statistically significant. SPSS version 12.0 (SPSS Inc., Chicago, IL, USA) was used for all statistical analyses.

\section{RESULTS}

A total of 601 patients were enrolled in our study, with 295 randomly assigned to the TT group and 306 to the ST group (Fig. 1).
Nineteen patients were lost to follow-up and one patient withdrew consent after receiving their eradication medicine. Among the remaining 581 patients, 22 were lost to follow-up, 12 took $<80 \%$ of their medication, and one withdrew consent. Therefore the final PP population consisted of 546 patients. Demographic and clinical characteristics at baseline were similar between the two treatment groups (Table 1).

In the ITT population, eradication rates were $70.8 \%$ in the TT group and $82.4 \%$ in the ST group ( $\mathrm{p}=0.001$ ) (Table 2). In the PP population, eradication rates were $76.9 \%$ with TT and $88.8 \%$ with ST $(\mathrm{p}=0.000)$. Bitter taste was the most common adverse effect (Table 3). The overall adverse effect rates were similar in the TT and ST groups (43.0\% vs $44.4 \%, \mathrm{p}=0.718$ ). Adherence to

Table 1. Baseline Characteristics of Patients

\begin{tabular}{lccc}
\multicolumn{1}{c}{ Variable } & Triple therapy $(\mathrm{n}=295)$ & Sequential therapy $(\mathrm{n}=306)$ & $\mathrm{p}$-value \\
\hline Age, yr & $54.07 \pm 12.6$ & $54.01 \pm 11.8$ & 0.955 \\
Male sex & $173(58.6)$ & $188(61.4)$ & 0.484 \\
Reason for eradication & & & $0.764^{*}$ \\
Gastric ulcer & 148 & 140 & 114 \\
Duodenal ulcer & 102 & 15 & 36 \\
Gastric+duodenal ulcer & 13 & 0 \\
Endoscopic treatment of EGCa & 29 & 1 \\
MALToma & 1 & 2 & \\
Others & 2 &
\end{tabular}

Data are presented as mean \pm SD or number $(\%)$.

EGCa, early gastric cancer; MALToma, mucosa-associated lymphoid tissue lymphoma.

${ }^{*}$ Fisher exact test.

Table 2. Eradication Rates and Compliance of Standard Triple Therapy and in Sequential Therapy

\begin{tabular}{llcc}
\hline & Triple therapy & Sequential therapy & p-value \\
\hline Eradication of first line treatment & & & \\
ITT & $209 / 295(70.8)$ & $252 / 306(82.4)$ & 0.001 \\
PP & $206 / 268(76.9)$ & $247 / 278(88.8)$ & 0.000 \\
\hline
\end{tabular}

Data are presented as number $(\%)$

ITT, intention-to-treat; PP, per protocol.

Table 3. Adverse Event Profiles of Triple Therapy and Sequential Therapy

\begin{tabular}{lccc}
\hline & Triple therapy & Sequential therapy & p-value \\
\hline Bitter taste & $81(28.5)$ & $72(24.2)$ & 0.242 \\
Nausea & $14(4.9)$ & $34(11.4)$ & 0.004 \\
Diarrhea & $34(12.0)$ & $22(7.4)$ & 0.062 \\
Headache & $2(0.7)$ & $8(2.7)$ & 0.065 \\
Epigastric discomfort & $8(2.8)$ & $11(3.7)$ & 0.548 \\
General weakness & $6(2.1)$ & $7(2.4)$ & 0.842 \\
Others & $9(3.1)$ & $13(4.4)$ & 0.446 \\
Any adverse events & $122(43.0)$ & $132(44.4)$ & 0.718 \\
Adherence & $268(98.5)$ & $278(97.2)$ & 0.280 \\
\hline
\end{tabular}

Data are presented as number (\%). 
the medication was also similar between the TT and ST groups (98.5\% vs $97.2 \%, p=0.280$ ).

\section{DISCUSSION}

ST demonstrated better eradication rates than TT in both ITT and PP analyses in the present nationwide study. Adverse events and treatment compliance were not statistically different between the two treatments. The efficacy of ST is challenged by metronidazole resistance and dual resistance to clarithromycin and metronidazole. ${ }^{24}$ In Korea, resistance to both antibiotics is high with resistance rates of $16.0 \%$ to $37.0 \%$ for clarithromycin and $35.8 \%$ to $56.3 \%$ for metronidazole. ${ }^{22,25,26}$ Dual resistance to clarithromycin and metronidazole is reported to be $3.2 \% .{ }^{25}$ The main reason that TT is still recommended as first-line therapy in Korea is because although ST achieved higher eradication rates than TT it did not reach optimal results in previous studies. ${ }^{23}$ The present nationwide multicenter study examined whether ST could replace TT as first-line therapy in Korea.

In the PP population, ST achieved eradication rates of $88.8 \%$ compared to $76.9 \%$ for TT. The absolute risk reduction was $12.0 \%$ (95\% confidence interval [CI], 5.7 to 18.2) and the number needed to treat was 9 (95\% CI, 5.5 to 18.2). These results indicate that ST can replace TT as first-line therapy in Korea. Another concern of ST is the development of antibiotic resistance and the choice of the second-line regimens after failure of ST. Several studies from Europe reported levofloxacin containing triple regimens to be effective as a second-line treatment. ${ }^{27-30}$ However, fluoroquinolone resistance is reported to be as high as $22.3 \%$ to $34.6 \%$ in Korea. ${ }^{22,25}$ Moxifloxacin containing TT following failed first-line ST achieved disappointing eradication rates of $60.4 \%$ and $70.7 \%$ for ITT and PP populations in Korea. ${ }^{31}$ Bismuth-based quadruple therapy (BQT) may be an alternative to levofloxacin-based regimens after failed ST. However, BQT should be used for 14 days after failed ST, since metronidazole resistance is suspected in these patients. ${ }^{32}$ Future studies regarding the optimal second line regimen after failure of ST may be needed before ST can be regarded as first line therapy.

TT is no longer effective in many parts of the world due to increasing clarithromycin resistance and has been deemed as an unethical comparator in clinical trials. ${ }^{12,33}$ However, a 7-day TT regimen is the only regimen reimbursed by the NHIS and is the most prescribed eradication regimen in Korea. A recent Korean study reported final PP eradication rates of TT to be $98.4 \%$ after second-line treatment and 99\% after third-line treatment. ${ }^{34} \mathrm{Al}$ though TT regimens may not achieve adequate eradication rates as first-line therapies, $H$. pylori infection was eradicated in most patients following second-line and third-line treatment. NonBQT such as ST and concomitant therapy may achieve higher eradication rates compared to TT as first-line therapy. However, it is doubtful that the ultimate eradication rates of these regimens will surpass that of TT. Currently most studies regarding first line therapy focus on the efficacy of a regimen as initial treatment. However, we believe that the cumulative eradication rates of a treatment are equally important. The use of non-BQT would expose patients to increased antibiotics which may result in increased antibiotic resistance after treatment. For these reasons, TT may be considered as a first-line regimen for $H$. pylori infection in Korea.

The main limitation of our study was the lack of evaluation of antibiotic resistance in the patients. However, antibiotic resistance testing is rarely performed before prescribing firstline regimens. So, the study mimics routine clinical practice. As mentioned above, 7-day TT is the most prescribe regimen in Korea and the only regimen reimbursed by the NHIS. Therefore, we chose this regimen as the comparator to ST; thus the duration of TT was shorter than ST. A recent meta-analysis reported that ST was superior to 7-day TT but was not significantly better than 14-day TT. ${ }^{35}$ Results of our study may have been different if the duration of TT was increased. Finally, the PPIs that were used in our study varied and this may have affected the eradication rates. We were not able to unify the PPIs because different institutions participated in our study. However, the efficacy of different PPIs for eradication have been reported to be similar in Korea and we believe the different PPIs would have a minimal effect on the eradication rates. ${ }^{36}$

In conclusion, this nationwide multicenter study suggests that ST achieves higher eradication rates than TT as first-line therapy in Korea. However, the PP eradication rates of the ST regimen was less than 90\% and little data exists regarding the optimal treatment regimen after failed ST. Further studies are needed to determine if it is worthwhile to include an additional antibiotic for first line therapy.

\section{CONFLICTS OF INTERESTS}

No potential conflict of interest relevant to this article was reported.

\section{ACKNOWLEDGEMENTS}

This study registered in Clinical Research Information Service (KCT0000378) and granted from Korean College of Helicobacter and Upper Gastrointestinal Research (2011-01).

\section{REFERENCES}

1. Blaser MJ. Helicobacter pylori and gastric diseases. BMJ 1998;316: 1507-1510.

2. Suerbaum S, Michetti P. Helicobacter pylori infection. N Engl J Med 2002;347:1175-1186.

3. Wang J, Xu L, Shi R, et al. Gastric atrophy and intestinal metaplasia before and after Helicobacter pylori eradication: a metaanalysis. Digestion 2011;83:253-260. 
4. Correa P, Fontham ET, Bravo JC, et al. Chemoprevention of gastric dysplasia: randomized trial of antioxidant supplements and antihelicobacter pylori therapy. J Natl Cancer Inst 2000;92:1881-1888.

5. Malfertheiner P, Sipponen P, Naumann M, et al. Helicobacter pylori eradication has the potential to prevent gastric cancer: a stateof-the-art critique. Am J Gastroenterol 2005;100:2100-2115.

6. Chan FK, Chung SC, Suen BY, et al. Preventing recurrent upper gastrointestinal bleeding in patients with Helicobacter pylori infection who are taking low-dose aspirin or naproxen. N Engl J Med 2001;344:967-973.

7. Chey WD, Wong BC; Practice Parameters Committee of the American College of Gastroenterology. American College of Gastroenterology guideline on the management of Helicobacter pylori infection. Am J Gastroenterol 2007;102:1808-1825.

8. Coelho LG, León-Barúa R, Quigley EM. Latin-American Consensus Conference on Helicobacter pylori infection: Latin-American National Gastroenterological Societies affiliated with the Inter-American Association of Gastroenterology (AIGE). Am J Gastroenterol 2000;95:2688-1691.

9. Malfertheiner P, Megraud F, O'Morain CA, et al. Management of Helicobacter pylori infection: the Maastricht IV/ Florence Consensus Report. Gut 2012;61:646-664.

10. Lam SK, Talley NJ. Report of the 1997 Asia Pacific Consensus Conference on the management of Helicobacter pylori infection. J Gastroenterol Hepatol 1998;13:1-12.

11. Gisbert JP, Calvet X. Review article: the effectiveness of standard triple therapy for Helicobacter pylori has not changed over the last decade, but it is not good enough. Aliment Pharmacol Ther 2011;34:1255-1268.

12. Graham DY, Fischbach L. Helicobacter pylori treatment in the era of increasing antibiotic resistance. Gut 2010;59:1143-1153.

13. Yim JY, Kim N, Choi SH, et al. Seroprevalence of Helicobacter pylori in South Korea. Helicobacter 2007;12:333-340.

14. Kim SG, Jung HK, Lee HL, et al. Guidelines for the diagnosis and treatment of Helicobacter pylori infection in Korea, 2013 revised edition. Korean J Gastroenterol 2013;62:3-26.

15. Gong EJ, Yun SC, Jung HY, et al. Meta-analysis of first-line triple therapy for helicobacter pylori eradication in Korea: is it time to change? J Korean Med Sci 2014;29:704-713.

16. Heo J, Jeon SW. Changes in the eradication rate of conventional triple therapy for Helicobacter pylori infection in Korea. Korean J Gastroenterol 2014;63:141-145.

17. Zullo A, Vaira D, Vakil N, et al. High eradication rates of Helicobacter pylori with a new sequential treatment. Aliment Pharmacol Ther 2003;17:719-726.

18. Kim JS, Ji JS, Choi H, Kim JH. Sequential therapy or triple therapy for Helicobacter pylori infection in Asians: systematic review and meta-analysis. Clin Res Hepatol Gastroenterol 2014;38:118-125.

19. Gatta L, Vakil N, Leandro G, Di Mario F, Vaira D. Sequential therapy or triple therapy for Helicobacter pylori infection: systematic review and meta-analysis of randomized controlled trials in adults and children. Am J Gastroenterol 2009;104:3069-3079
20. Jafri NS, Hornung CA, Howden CW. Meta-analysis: sequential therapy appears superior to standard therapy for Helicobacter pylori infection in patients naive to treatment. Ann Intern Med 2008;148:923-931.

21. Tong JL, Ran ZH, Shen J, Xiao SD. Sequential therapy vs. standard triple therapies for Helicobacter pylori infection: a metaanalysis. J Clin Pharm Ther 2009;34:41-53.

22. Lee JW, Kim N, Kim JM, et al. Prevalence of primary and secondary antimicrobial resistance of Helicobacter pylori in Korea from 2003 through 2012. Helicobacter 2013;18:206-214.

23. Kim JS, Kim BW, Ham JH, et al. Sequential therapy for Helicobacter pylori infection in Korea: systematic review and metaanalysis. Gut Liver 2013;7:546-551.

24. Graham DY, Lee YC, Wu MS. Rational Helicobacter pylori therapy: evidence-based medicine rather than medicine-based evidence. Clin Gastroenterol Hepatol 2014;12:177-186.e3.

25. An B, Moon BS, Kim H, et al. Antibiotic resistance in Helicobacter pylori strains and its effect on $\mathrm{H}$. pylori eradication rates in a single center in Korea. Ann Lab Med 2013;33:415-419.

26. Park CS, Lee SM, Park CH, et al. Pretreatment antimicrobial susceptibility-guided vs. clarithromycin-based triple therapy for Helicobacter pylori eradication in a region with high rates of multiple drug resistance. Am J Gastroenterol 2014;109:1595-1602.

27. Gisbert JP, Pérez-Aisa A, Bermejo F, et al. Second-line therapy with levofloxacin after failure of treatment to eradicate helicobacter pylori infection: time trends in a Spanish Multicenter Study of 1000 patients. J Clin Gastroenterol 2013;47:130-135.

28. Gisbert JP, Bermejo F, Castro-Fernández M, et al. Second-line rescue therapy with levofloxacin after $\mathrm{H}$. pylori treatment failure: a Spanish multicenter study of 300 patients. Am J Gastroenterol 2008;103:71-76.

29. Manfredi M, Bizzarri B, de'Angelis GL. Helicobacter pylori infection: sequential therapy followed by levofloxacin-containing triple therapy provides a good cumulative eradication rate. Helicobacter 2012;17:246-253.

30. Zullo A, De Francesco V, Manes G, Scaccianoce G, Cristofari F, Hassan C. Second-line and rescue therapies for Helicobacter pylori eradication in clinical practice. J Gastrointestin Liver Dis 2010;19: 131-134.

31. Kang KK, Lee DH, Oh DH, et al. Helicobacter pylori eradication with moxifloxacin-containing therapy following failed first-line therapies in South Korea. World J Gastroenterol 2014;20:69326938.

32. Wu JY, Liou JM, Graham DY. Evidence-based recommendations for successful Helicobacter pylori treatment. Expert Rev Gastroenterol Hepatol 2014;8:21-28.

33. Graham DY, Fischbach LA. Letter: the ethics of using inferior regimens in $\mathrm{H}$. pylori randomised trials. Aliment Pharmacol Ther 2012;35:852-854.

34. Yoon K, Kim N, Nam RH, et al. Ultimate eradication rate of Helicobacter pylori after first, second, or third-line therapy in Korea. J Gastroenterol Hepatol 2015;30:490-495. 
Kim JS, et al: Sequential Therapy versus Triple Therapy in Korea 561

35. Gatta L, Vakil N, Vaira D, Scarpignato C. Global eradication rates for Helicobacter pylori infection: systematic review and metaanalysis of sequential therapy. BMJ 2013;347:f4587.

36. Keum B, Lee SW, Kim SY, et al. Comparison of Helicobacter pylori eradication rate according to different PPI-based triple therapy: omeprazole, rabeprazole, esomeprazole and lansoprazole. Korean J Gastroenterol 2005;46:433-439. 